\title{
Synthesis of PMMA-co-PMAA Copolymer Brush on Multi-Wall Carbon Nanotubes
}

\author{
Petr Slobodian ${ }^{1,2 *}$, Petr Svoboda ${ }^{2,3}$, Pavel Riha ${ }^{4}$, Roman Boruta ${ }^{1}$, Petr Saha ${ }^{1,2}$ \\ ${ }^{1}$ Polymer Centre, Faculty of Technology, Tomas Bata University, Zlin, Czech Republic; ${ }^{2}$ Centre of Polymer Systems, University \\ Institute, Tomas Bata University, Zlin, Czech Republic; ${ }^{3}$ Department of Polymer Engineering, Faculty of Technology, Tomas Bata \\ University, Zlin, Czech Republic; ${ }^{4}$ Institute of Hydrodynamics, Academy of Sciences, Prague, Czech Republic. \\ Email: "slobodian@ft.utb.cz
}

Received May $15^{\text {th }}$, 2012; revised June 21 ${ }^{\text {st }}$, 2012; accepted June $30^{\text {th }}, 2012$

\begin{abstract}
Poly(methyl methacrylate)/poly(methacrylamide) copolymer (PMMA-co-PMAA) was synthesized by a free radical copolymerization of MMA and MAA monomers in methylethyl ketone using AIBN as radical initiator. Multi-wall carbon nanotubes (MWCNT) were oxidized in $\mathrm{KMnO}_{4}$ acidic suspension. Carboxyl groups on the surface oxidized MWCNT were reacted with primary amide group of PMMA-co-PMAA copolymer in MEK solution under ultrasound to form polymer brush on the surface of MWCNT. With the help of TG analyses the amount of covalently grafted PMMA-co-PMAA copolymer onto MWCNT surface was determined as $~ 47 \mathrm{wt} \%$. TEM analyses identified thin copolymer layer adhered onto MWCNT surface with average thickness $\sim 5 \mathrm{~nm}$.
\end{abstract}

Keywords: Carbon Nanotubes; Functionalization; PMMA; PMAA; Polymer Brush

\section{Introduction}

Carbon nanotubes, CNT, are considered as very prospective filler for polymeric composite material materials. Although they have been already practically used, substantial technological problems remain. At first, it is very problematic to disperse CNT in a polymeric matrix due to their insolubility in organic materials and also due to strong tendency to agglomerate [1-3]. Another critical point referring to polymeric/CNT composites seems to be the issue of a good adhesion between a polymeric matrix and CNT surface. Generally, their good compatibility is not expected [4,5] although some experimental works disprove this $[6,7]$.

Therefore the development and innovation of CNT/ polymeric nanocomposites preparation methods is still a current issue. Commonly used methods, such as mixing in the polymeric melt, are being replaced by more efficient methods using e.g. ultrasound as a stirring element. Other efficient way to improve CNT dispersion in the polymeric matrix and to increase transfer of tension through polymer/CNT interface is chemical functionalisation of CNT when covalent bond of organic molecules or polymeric fragments on CNT surface is being created. Probably the most progressive method seems to be the process of CNT oxidation [8,9] leading to creation of

${ }^{*}$ Corresponding author. carboxyl and hydroxyls groups which become a binding element for different polymeric molecules [10-14].

Copolymers of methyl methacrylate (MMA) with methacrylamide (MAA) (PMMA-co-PMAA) can be considered as high glass transition copolymers of poly(methyl methacrylate) (PMMA). Their improved thermal properties are due to strong hydrogen bonding interactions between this two types of monomer segments $[15,16]$. These copolymers also pose high degree of homogeneity caused by a good miscibility [16] high transparency or improved adhesion to substrates like silicate glass [17]. Their practical importance is for example in area of optical-electronic industry. One possible way how to prepare PMMA-co-PMAA copolymers is a free radical copolymerization of appropriate monomers in a solvent. As it was shown [15-17] the copolymer composition depends on initial monomers MMA:MAA ratio. At a low MMA concentration in monomer mixture the content of monomer units in copolymer is only slightly higher but at higher content of MAA the product is enriched in MAA units rendering isolated PMAA units along copolymer main-chain [15].

The aim of the present paper is to show a possibility how to fabricate polymer brush of PMMA copolymer onto a surface of oxidized MWCNT. Such surface treated MWCNT filler can be further used in fabrication of polymer/MWCNT nanocomposites. As it is usually shown, 
the polymer layer adhered onto CNT material leads to better deagglomeration of MWCNT bundles and more uniform dispersion of MWCNT through polymer matrix, together with better transfer of load from polymer to tubes. The prepared MWCNT-g-(PMMA-co-PMAA) material can be for example used as filler into pure commercial PMMA matrix. In this system better adhesion of PMMA-co-PMAA copolymer brush to PMMA matrix due to strong hydrogen bonding interaction between PMMA and PAAM can be expected compared to the case of pure PMMA.

\section{Experimental}

Composite preparation: Methyl methacrylate (MMA) and methacrylamide (MAA) monomers, Azobisisobutyronitrile (AIBN) a free radical initiator, methylethyl ketone (MEK) a solvent, Potassium permanganate $\left(\mathrm{KMnO}_{4}\right)$ an oxidizing agent were purchased from Aldrich. The Multi-wall carbon nanotubes (MWCNT), acetylene type, purified, were supplied by Sun Nanotech Co. Ltd., China (diameter $10-30 \mathrm{~nm}$, length $1-10 \mathrm{~m}$, purity $>90 \%$ and volume resistivity $0.12 \Omega \cdot \mathrm{cm}$ reported by supplier) and were used as received.

A solution radical polymerization of MMA and copolymerization of MMA with MAA were performed in a $40 \mathrm{wt} \%$ solution [17] of monomers in MEK $(60 \mathrm{ml})$ at $70^{\circ} \mathrm{C}$ for 20 hours using AIBN $(0.16 \mathrm{~g})$ as initiator. The molar concentration of MAA in the reaction mixture was 9 mol\%. Finally, solution radical polymerization of pure MAA was done at identical condition with monomer concentration of $25 \mathrm{wt} \%$ in MEK. The synthesis was performed in the three neck glass reactor $\left(500 \mathrm{~cm}^{3}\right)$ fitted with a mechanical stirrer, a reflux condenser and nitrogen inlet. The precise temperature was controlled by thermostatic bath Julabo HP4.

MWCNT crude material was oxidized by $\mathrm{KMnO}_{4}$ [8]. Into a glass reactor with a reflux condenser filled with $250 \mathrm{~cm}^{3}$ of $0.5 \mathrm{M} \mathrm{H}_{2} \mathrm{SO}_{4}, 5 \mathrm{~g}$ of $\mathrm{KMnO}_{4}$ and $2 \mathrm{~g}$ of MWCNT were added. Then the dispersion was sonicated using thermostatic ultrasonic bath (Bandelin electronic DT $103 \mathrm{H}$ ) at $85^{\circ} \mathrm{C}$ for 15 hours. The product was filtered and washed with concentrated $\mathrm{HCl}$ to remove the $\mathrm{MnO}_{2}$ followed by washing with deionized (DI) water and dried.

The MWCNT nanocomposite with copolymer matrix (PMMA-co-PMAA) was prepared by sonication of prepared copolymer solution together with oxidized CNT (1 wt\% of MWCNT-ox. per total copolymer weight) using Dr. Hielscher GmbH apparatus with ultrasonic horn, S7, for 3 hours at $60^{\circ} \mathrm{C}$. Prepared CNT dispersion in copolymer as well as other prepared polymer/MEK solutions were precipitated by pouring into RT, then it was water filtered and dried. The products were used for following calorimetric and FT-IR analyses. The additional specimen for TG and TEM analyses was prepared to investigate the amount of PMMA-co-PMAA copolymer covalently linked onto the surface of MWCNT material denoted as MWCNT-g-(PMMA-co-PMAA), where $g$ means "grafted". The prepared CNT dispersion in MEK solution of PMMA-co-PMAA was centrifuged at 6000 rpm for $15 \mathrm{~min}$. The black-colored supernatant with MWCNT- $g$-(PMMA-co-PMAA) particles was decanted and refluxed in access of MEK for 30 min to dissolve un-grafted polymer (concentration of particles in MEK was around $0.1 \mathrm{wt} \%)$ then centrifuged, decanted and again refluxed.

Analyses: Differential scanning calorimetry, DSC (Perkin-Elmer DSC 1 Pyris) was used to measure glass transition temperature, $T_{g}$. The temperature and heat flow of the apparatus was calibrated on indium standard. Temperature program was selected as follows: annealing at $280^{\circ} \mathrm{C}$ for $3 \mathrm{~min}$, then rapid cooling to $30^{\circ} \mathrm{C}$ and subsequently reheating thought $T_{g}$ region by heating rate $20^{\circ} \mathrm{C} / \mathrm{min}, T_{g}$ was evaluated as inflection point. Thermogravimetric analyses (TGA) of the samples were studied using thermogravimeter SETARAM SETSYS Evolution 1200. The samples were examined under inert atmosphere of helium (5.5 purity, SIAD TP); the gas flow was $30^{\circ} \mathrm{cm}^{3} / \mathrm{min}$ at the pressure of $101.325 \mathrm{kPa}$ (i.e. $30 \mathrm{sccm}$ ) for all experiments. A platinum crucible was used for the sample, the weight of which was about $4 \mathrm{mg}$. Temperature was increased at the rate of $20^{\circ} \mathrm{C} / \mathrm{min}$, in the range from the ambient temperature up to $1200^{\circ} \mathrm{C}$ for non-isothermal analysis. FT-IR spectra were recorded on a Nicolet Avatar 320 FT-IR spectrometer collected after 32 scans with spectral resolution of $1 \mathrm{~cm}^{-1}$. The structure of refluxed MWCNT-g-(PMMA-co-PMAA) nanocomposite was analyzed using transmission electron microscopy, TEM. Samples for TEM were prepared using 300 mesh cooper grids with holey carbon film (SPI, USA) and examined under the transmission electron microscope JEOL JEM 2010 at the accelerating voltage $160 \mathrm{kV}$.

\section{Results and Discussion}

The complete synthetic sequence from oxidation of MWCNT with $\mathrm{KMnO}_{4}$, preparation of PMMA-co-PMAA copolymer to the possible scenario of grafting of PMMAco-PMAA molecules onto surface of oxidized MWCNTox. are illustrated at Schemes 1(a)-(c). Part (a) represents the MWCNT surface-modification reaction with $\mathrm{KMnO}_{4}$. The color of CNT dispersion was changed from dark purple to dark brown as $\mathrm{Mn}^{7+}$ forms are reduced to $\mathrm{Mn}^{4+}$ ones. Part (b) denotes radical copolymerization of MMA and MAA. Our experimentally prepared PMMA-coPMAA solution in MEK was homogenous and transparent. Finally, it is necessary to denote that intensive mechanical stirring during polymerization is necessary to 
restrain creation of bimodal product or even precipitation of PMAA-reach copolymer from reaction mixture. The possible covalent bonding between copolymer and functionalized nanotubes presents part (c) of Scheme 1. The primary amide groups of copolymer link to the carboxylic groups attached to the nanotube forming imide covalent bonds. Such possible reaction way was also outlined in other systems. Such as for example reaction of $\left(\mathrm{NH}_{2}\right)$ group of amide functionalized SWCNT with PMMA via side chain $\left(\mathrm{COOCH}_{3}\right)$ leading also to amide covalent bonds (in N-methyl pyrrolidone by sonication) [10] or by reaction of COOH-functionalized MWCNT with melamine-formaldehyde resin containing free amino groups [19]. The product of this reaction was black longtime stable suspension. Their centrifugation under above described condition leads only to black supernatant and also black copolymer solution above it containing probably better separated CNT.

For next analyses the polymerization products were precipitated by pouring into RT water. DSC analyses of precipitated copolymer pose single $T_{g}$ at $128.8^{\circ} \mathrm{C} \pm 0.8^{\circ} \mathrm{C}$ indicating that the product is homogenous. There was detected no significant change in $T_{g}$ value by incorporating oxidized CNT into PMMA-co-PMAA copolymer in the range of measurement's error. Finally, also glass transition temperatures for homopolymers were determined as $T_{g}=105.2^{\circ} \mathrm{C} \pm 0.7^{\circ} \mathrm{C}$ for neat PMMA (very similar to usually published value for PMMA $[15,16,18])$ and $T_{g}=$ $213^{\circ} \mathrm{C} \pm 4^{\circ} \mathrm{C}$ for PMAA (available literature data for PMAA significantly vary such as for example $170^{\circ} \mathrm{C}$ [16] or $251^{\circ} \mathrm{C}$ [15]). Noticed increase in copolymer's $T_{g}$ compared to pure PMMA is usually explained as influence of polar MAA units when strong intermolecular interactions between MAA and MMA units occurs due to the formation of hydrogen bonds [15-17].

The content of PMMA-co-PMAAM copolymer grafted onto the surface of MWCNT was determined with the help of thermogravimetric analyses (TGA). Figure 1 shows TGA curves of the four studied samples. Line (a) shows the pure MWCNT as-received material; (b) is for oxidized MWCNT by $\mathrm{KMnO}_{4}$ (MWCNT-ox.); (c) is for pure PMMA-co-PMAAM copolymer and (d) represents MWCNT- $g$-(PMMA-co-PMAAM) nanocomposite. As can be seen, MWNT shows hardly any degradation in the used range of heating, here displayed up to $700^{\circ} \mathrm{C}$; only very small mass loss of ca $3 \mathrm{wt} \%$ at the $700^{\circ} \mathrm{C}$ was observed. This decomposition is probably caused by amorphous carbon contained in the original material together with decomposition of functional groups like $\mathrm{O}-\mathrm{C}=\mathrm{O}$ or C-O also included in crude material [19]. On the other hand, oxidized tubes, MWCNT-ox., shows slightly higher weight loss achieving at mentioned $700^{\circ} \mathrm{C}$ about ca 6 wt\%. This increase is probably due to higher content of functional groups, mainly acidic sites (but also -OH or $\mathrm{C}=\mathrm{O}$ groups [20]) which should be expected to be introduced during oxidizing process [8]. Also a small amount of amorphous carbon after the $\mathrm{KMnO}_{4}$ oxidation process can be expected [8] although other report shows that oxidation by $\mathrm{KMnO}_{4}$ in an acidic suspension provides nanotubes free of amorphous carbon [9]. Trace for PMMA-co-PMAAM (line c) clearly shows several decomposition events, similar to [17]. The first one is located at ca. $180^{\circ} \mathrm{C}$ (8 wt $\%$ mass loss), next smaller one at ca $280^{\circ} \mathrm{C}$ (6 wt $\%$ mass loss) both followed by main decomposition of PMMA-co-PMAAM main chain. It starts at ca $370^{\circ} \mathrm{C}$ with maximal rate at ca $428^{\circ} \mathrm{C}$ and is totally

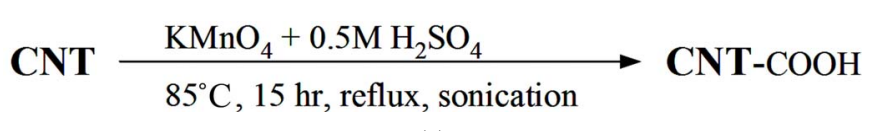

(a)<smiles>C=C(C)C(N)=[C+]CC(C)C(=O)Cl</smiles><smiles>COC(=O)C(C)CC(C)(C[C@@H](O)C#[W])C(N)=O</smiles>

(c)

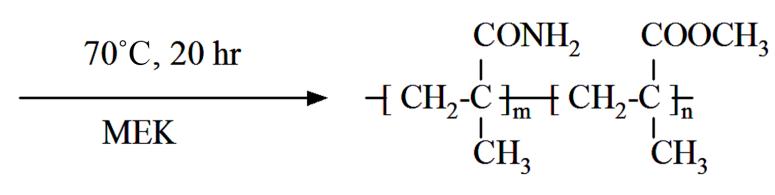

(b)<smiles>CCC(C)(CC(C)C(=O)OC)C(=O)N[Al]</smiles>

Scheme 1. Synthetic route used to prepare MWCNT-grafted-(PMMA-co-PMAAM) nanocomposite. (a) Oxidation of MWCNT using $\mathrm{KMnO}_{4}$ to increase content of carboxyl groups; (b) Copolymerization of MMA and MAAM monomers; (c) Functionalization of oxidized CNT to prepare MWCNT-g-(PMMA-co-PMAAM) nanocomposite. 
decomposed at $\sim 460^{\circ} \mathrm{C}$. Total mass loss of each events is ca 96 wt\%. In MWCNT-g-(PMMA-co-PMAAM) nanocomposite (line d) the main decomposition events starts at the same temperatures as for PMMA-co-PMAAM, goes thought the maximal decomposition rate at $426^{\circ} \mathrm{C}$ to be totally decomposed again at $\sim 460^{\circ} \mathrm{C}$. The amount of PMMA-co-PMAAM covalently grafted onto surface of MWCNT in MWCNT-g-(PMMA-co-PMAAM) nanocomposite was determined as the difference in residual weight between PMMA-co-PMAAM, MWNT-ox. and MWCNT-g-(PMMA-Co-PMAAM) at $460^{\circ} \mathrm{C}$ to be $42 \mathrm{wt} \%$. The calculated value is comparable with values usually found for polymer grafted CNT composites. For example $24 \mathrm{wt} \%$ of PS using silane-coupling agent [11] or 37 wt $\%$ of PS for MWCNT- $g$-PS composite prepared by radical coupling via in situ bulk polymerization [21], or $45 \mathrm{wt} \%$ of PS by technique when polymerization capable vinyl groups are incorporating onto CNT surface (preparation of so called CNT-mer) [12].

The chemical structure of prepared polymeric materials can be investigated with the help of FT-IR spectroscopy. Figure 2 shows scale-expanded infrared spectra of pure PMMA, pure PMAA, PMMA-co-PMAA copolymer and MWCNT-g-(PMMA-co-PMAA) nanocomposite in the range $1550-1800 \mathrm{~cm}^{-1}$. Pure PMMA shows band at $1722 \mathrm{~cm}^{-1}$ corresponding to carbonyl stretching of free carbonyl groups [10,15-17]. Pure PMAA shows two bands at $1652 \mathrm{~cm}^{-1}$ and $1597 \mathrm{~cm}^{-1}$ corresponding to the amide $I$ band $(\mathrm{C}=\mathrm{O}$ stretching) and amide band $(\mathrm{N}-\mathrm{H}$ bending), respectively [15]. The amide band has an intensity of one-half of that of the carbonyl $I$ absorption band. In PMMA-co-PMAA copolymer, the absorption of the both amide $I$ and amide band are clearly shifted to higher wavenumber about $1679 \mathrm{~cm}^{-1}$ and $1605 \mathrm{~cm}^{-1}$ caused by hydrogen-bonding interaction between the carbonyl groups of PMMA and the amide group of PMAA [15]. A FT-IR spectrum of synthesized nanocompozite MWCNT-g-(PMMA-co-PMAA) shows three bands at 1721,1663 and $1590 \mathrm{~cm}^{-1}$. New covalent bond between CNT and copolymer is according reaction Scheme 1 functional imide group (-CO-NH-CO-) which carbonyl group absorbs at $1720 \mathrm{~cm}^{-1}$ [22] so at the same position as $(\mathrm{C}=\mathrm{O})$ from PMMA. The relative intensity of PMMA band in MWCNT-g-(PMMA-Co-PMAA) is diminished compared to PMAA amide bands compared to pure PMMA-co-PMAA copolymer. Amide I band is shifted to lower wavenumber but it is still higher than in pure PMAA. It was found [15] that amide $I$ group is clearly shifted to higher wavenumber with increasing PMMA content. So it seems that PMMA-co-PMAA copolymer layer grafted onto surface of CNT is enriched by PMAA sequences compared to pure PMMA-co-PMAA prepared copolymer.

The TEM images of MWCNT- $g$-(PMMA-co-PMAAM) nanocomposite are shown in Figures 3(a) and (b)). The TEM micrographs clearly confirm the presence of PMMAco-PMAAM copolymer layer adhering onto the MWCNT surface. Here two different shells can be observed. The first represents core-shell structure of MWCNT with whole diameter $\sim 20 \mathrm{~nm}$ which is assembled from $\sim 12$ of individual graphitic layers with distance between particular layer $\sim 0.35 \mathrm{~nm}$. The second is created from PMMA-coPMAAM copolymer with thickness $\sim 5 \mathrm{~nm}$. The different studies shows very similar thickness of polymer layers adhered onto CNT surface such as for example $4.0 \mathrm{~nm}$ [12], $5.0 \mathrm{~nm}$ [11] or for $7-10 \mathrm{~nm}$ [23].

\section{Conclusion}

Functionalized multi wall carbon nanotubes covered by

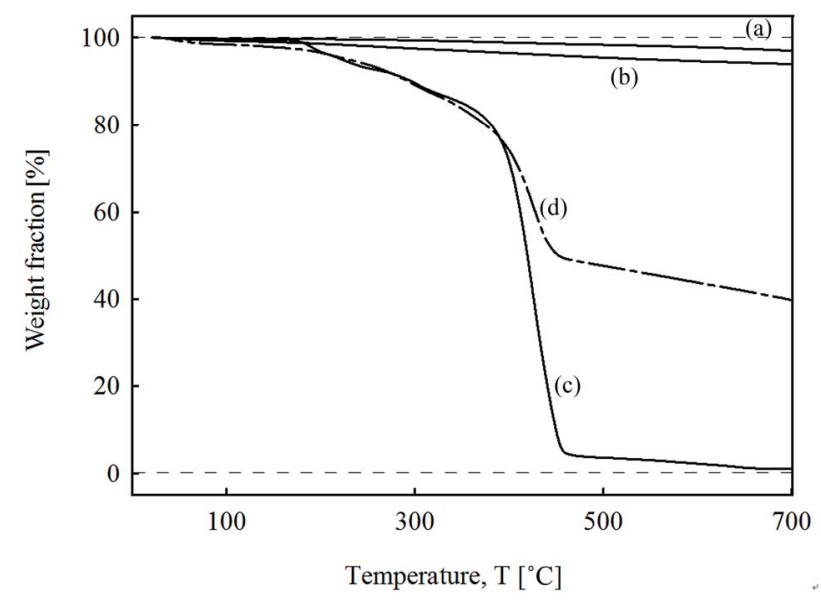

Figure 1. Thermogravimetric analysis of as-received MWCNT (a); $\mathrm{KMnO}_{4}$ oxidized MWCNT-ox (b); PMMA-co-PMAAM copolymer (c); and MWCNT-g-(PMMA-co-PMA-AM) nanocomposite (d).

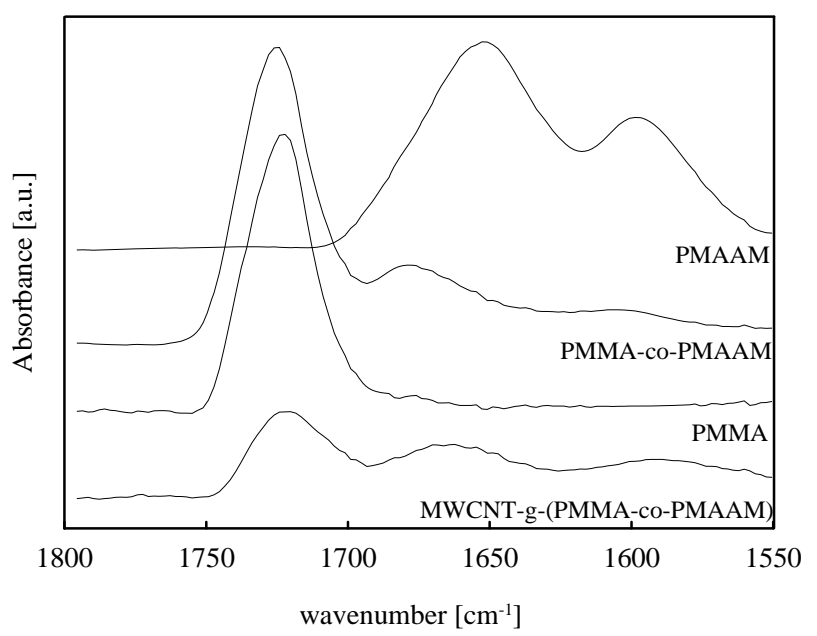

Figure 2. The IR spectra for homopolymers PMMA and PMAA, copolymer PMMA-co-PMAAM and MWCNT-g(PMMA-co-PMAAM) nanocomposite at $1550-1800 \mathrm{~cm}^{-1}$ at room temperature. 


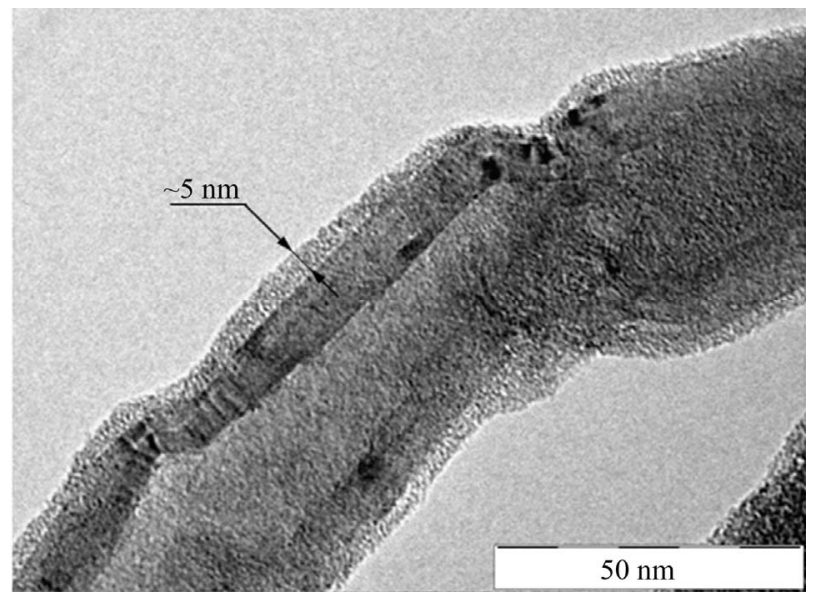

(a)

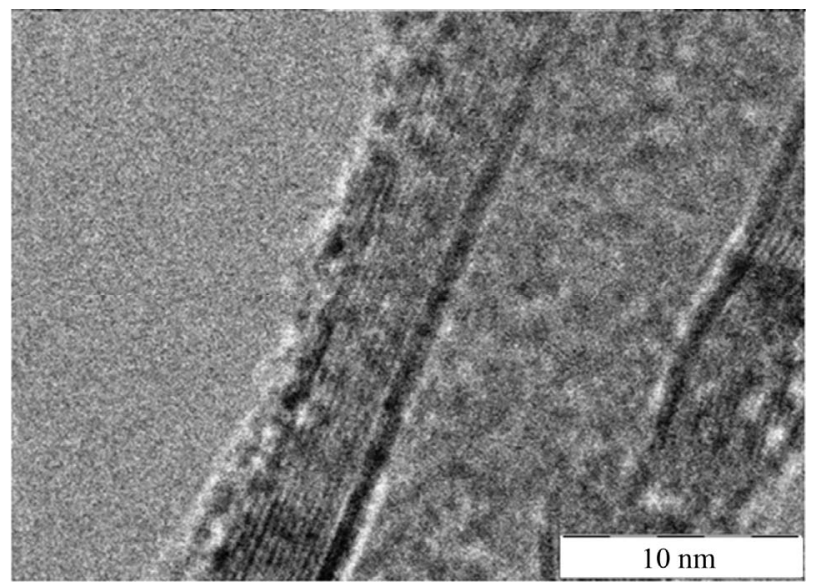

(b)

Figure 3. TEM morphology of MWCNT-g-(PMMAco-PMAAM) nanocomposite.

PMMA-co-PMAA copolymer brush were successfully synthesized by reaction between carboxylated MWCNT with activated primary amide group of PMMA-co-PMAA copolymer. The FT-IR spectra indicate the interaction present in MWCNT-g-(PMMA-co-PMAAM) nanocomposite. The amount of covalently attached copolymer onto surface of MWCT was determined with the help of TGA analysis. It reaches about $47 \mathrm{wt} \%$ with average thickness of copolymer brush adhered onto MWCNT surface of about $5 \mathrm{~nm}$ analyzed by TEM. PMMA-coPMAA copolymer functionalized MWCNT filler may be promising filler in application such as polymer/carbon nanotube composites acting as more effective reinforcing filler compared to crude CNT material. PMMA polymer matrix can by suitable when additional strong hydrogen bonding interaction between matrix and PMMA-co-PMAA brush can be expected.

\section{Acknowledgements}

The work was supported by the Operational Program of
Research and Development for Innovations co-funded by the European Regional Development Fund (ERDF), the National budget of Czech Republic within the framework of the Centre of Polymer Systems project (Reg. No.: CZ.1.05/2.1.00/03.0111). This article was also supported by the internal grant of TBU in Zlin No. IGA/FT/2012/ 022 funded from the resources of Specific University Research.

\section{REFERENCES}

[1] H. T. Ham, Y. S. Choi and I. J. Chung, “An Explanation of Dispersion States of Single-Walled Carbon Nanotubes in Solvents and Aqueous Surfactant Solutions Using Solubility Parameters," Journal of Colloid and Interface Science, Vol. 286, No. 1, 2005, pp. 216-223. doi:10.1016/j.jcis.2005.01.002

[2] K. A. Narh, L. Jallo and K. Y. Rhee, "The Effect of Carbon Nanotube Agglomeration on the Thermal and Mechanical Properties of Polyethylene Oxide,” Polymer Composites, Vol. 29, No. 7, 2008, pp. 809-817. doi:10.1016/j.jcis.2005.01.002

[3] P. Slobodian, A. Lengálová, M. Šlouf and P. Sáha, "Poly (Methyl Methacrylate)/Multi-Wall Carbon Nanotubes Composites Prepared by Solvent Cast Technique: Composites Electrical Percolation Threshold," Journal of Reinforced Plastics and Composites, Vol. 26, No. 16, 2007, pp. 17051712. doi:10.1177/0731684407081437

[4] K. Schulte and A. H. Windle, "Special Issue: Carbon Nanotube (CNT)-Polymer Composites,” Composites Science and Technology, Vol. 67, No. 5, 2007, p. 777. doi:10.1016/j.compscitech.2006.08.020

[5] L. S. Schadler, S .C. Giannaris and P. M. Ajayan, "Load Transfer in Carbon Nanotube Epoxy Composites," Applied Physics Letters, Vol. 73, No. 26, 1998, pp. 38423444. doi:10.1063/1.122911

[6] H. D. Wagner, "Nanotube-Polymer Adhesion: A Mechanics Approach," Chemical Physics Letters, Vol. 361, No. 1-2, 2002, pp. 57-61. doi:10.1016/S0009-2614(02)00948-X

[7] M. Wong, M. Paramsothy, X. J. Xu, Y. Ren, S. Li and K. Liao, "Physical Interactions at Carbon Nanotube-Polymer Interface,” Polymer, Vol. 44, No. 25, 2003, pp. 7757 7764. doi:10.1016/j.polymer.2003.10.011

[8] A. Rasheed, J. Y. Howe, M. D. Dadmun and P. F. Britt, "The Efficiency of the Oxidation of Carbon Nanofibers with Various Oxidizing Agents,” Carbon, Vol. 45, No. 5, 2007, pp. 1072-1080. doi:10.1016/j.carbon.2006.12.010

[9] K. Hernadi, A. Siska, L. Thien-Nga, L. Forro and I. Kiricsi, "Reactivity of Different Kinds of Carbon during Oxidative Purification of Catalytically Prepared Carbon Nanotubes,” Solid State Ionics, Vol. 141, 2001, pp. 203-209. doi:10.1016/S0167-2738(01)00789-5

[10] T. Ramanathan, H. Liu, L. C. Brinson and J. Polym, "Functionalized SWNT/Polymer Nanocomposites for Dramatic Property Improvement,” Journal of Polymer Science Part B: Polymer Physics, Vol. 43, No. 17, 2005, pp. 2269-2279. doi:10.1002/polb.20510 
[11] M. H. Kim, C. K. Hong, S. Choe and S. E. Shim, "Synthesis of Polystyrene Brush on Multiwalled Carbon Nanotubes Treated with $\mathrm{KMnO}_{4}$ in the Presence of a PhaseTransfer Catalyst," Journal of Polymer Science Part A: Polymer Chemistry, Vol. 45, No. 19, 2007, pp. 4413-4420. doi:10.1002/pola.22190

[12] J. U. Ha, M. Kim, J. Lee, S. Choe, I. W. Cheong, S. E. Shim, "A Novel Synthesis of polymer Brush on Multiwall Carbon Nanotubes Bearing Terminal Monomeric Unit," Journal of Polymer Science Part A: Polymer Chemistry, Vol. 44, 2006, pp. 6394-6401. doi:10.1002/pola.22190

[13] J. W. Xiong, Z. Zheng, X. M. Qin, M. Li, H. Q. Li and X. L. Wang, "The Thermal and Mechanical Properties of a Polyurethane/Multi-Walled Carbon Nanotube Composite,” Carbon, Vol. 44, No. 13, 2006, pp. 2701-2707. doi:10.1016/j.carbon.2006.04.005

[14] T. Ramanathan, F. T. Fisher, R. S. Ruoff and L. C. Brinson, "Amino-Functionalized Carbon Nanotubes for Binding to Polymers and Biological Systems," Chemistry of Materials, Vol. 17, No. 6, 2005, pp. 1290-12958. doi:10.1016/j.carbon.2006.04.005

[15] S. W. Kuo, H. C. Kao and F. C. Chang, "Thermal Behavior and Specific Interaction in High Glass Transition Temperature PMMA Copolymer,” Polymer, Vol. 44, No. 22, 2003, pp. 6873-6882. doi:10.1016/j.polymer.2003.08.026

[16] C. F. Huang and F. C. Chang, "Comparison of Hydrogen Bonding Interaction between PMMA/PMAA Blends and PMMA-co-PMAA Copolymers,” Polymer, Vol. 44, No. 10, 2003, pp. 2965-2974. doi:10.1016/j.polymer.2003.08.026

[17] E. O. Kazyuka, A. P. Polikarpov, I. G. Poplevka, Y. I. Matusevich, L. B. Yakitntsova and L .P. Krul, “Thermal
Properties of Copolymerization Products of Methyl Methacrylate with Methacrylamide," Russian Journal of Applied Chemistry, Vol. 80, No. 11, 2007, pp. 1898-1901. doi:10.1134/S1070427207110237

[18] J. Brandrup and E. H. Immegut, "Polymer Handbook," Wiley \& Sons Inc., New York, 1989.

[19] L. Licea-Jimenez, P.Y. Henrio, A. Lund, T. M. Laurie, S. A. Perez-Garcia, L. Nyborg, H. Hassander, H. Bertilsson and R. W. Rychwalski, "MWNT Reinforced MelamineFormaldehyde Containing Alpha-Cellulose,” Composites Science and Technology, Vol. 67, No. 5, 2007, pp. 844854. doi:10.1016/j.compscitech.2006.01.031

[20] J. Zhang, H. L. Zou, Q. Qing, Y. L. Yang, Q. W. Li, Z. F. Liu, X. Y. Guo and Z. L. Du, "Effect of Chemical Oxidation on the Structure of Single-Walled Carbon Nanotubes," Journal of Physical Chemistry B, Vol. 107, No. 16, 2003, pp. 3712-3718. doi:10.1016/j.compscitech.2006.01.031

[21] S. T. Kim, H. J. Choi and S. M. Hong, "Bulk Polymerized Polystyrene in the Presence of Multiwalled Carbon Nanotubes,” Colloid and Polymer Science, Vol. 285, No. 5, 2007, pp. 593-598. doi:10.1016/j.compscitech.2006.01.031

[22] Y. Wang, S. H. Goh and T. S. Chung, "Miscibility Study of Torlon ${ }^{\circledR}$ Polyamide-Imide with Matrimid ${ }^{\circledR} 5218$ Polyimide and Polybenzimidazole,” Polymer, Vol. 48, No. 10, 2007, pp. 2901-2909. doi:10.1016/j.polymer.2007.03.040

[23] Y. Yang, X. Wang, L. Liu, X. Xie, Z. Yang, R. K. Y. Li and Y. Mai, "Structure and Photoresponsive Behaviors of Multiwalled Carbon Nanotubes Grafted by Polyurethanes Containing Azobenzene Side Chains,” Journal of Physical Chemistry C, Vol. 111, No. 30, 2007, pp. 11231-11239. doi:10.1021/jp0728510 